PROCEEDINGS OF THE

AMERICAN MATHEMATICAL SOCIETY

Volume 133, Number 7, Pages 1887-1890

S 0002-9939(05)07827-5

Article electronically published on February 15, 2005

\title{
FIBONACCI NUMBERS THAT ARE NOT SUMS OF TWO PRIME POWERS
}

\author{
FLORIAN LUCA AND PANTELIMON STĂNICĂ
}

(Communicated by David E. Rohrlich)

\begin{abstract}
In this paper, we construct an infinite arithmetic progression $\mathcal{A}$ of positive integers $n$ such that if $n \in \mathcal{A}$, then the $n$th Fibonacci number is not a sum of two prime powers.
\end{abstract}

\section{INTRODUCTION}

In 1849, A. de Polignac [7] asked if every odd positive integer can be represented as the sum of a power of 2 and a prime (or 1). Euler did note that 959 was not of this form. Romanoff [8] used the Brun sieve to show that a positive proportion of integers are representable in this way. Later, van der Corput 2] showed that a positive proportion of integers are not representable in this way by using covering congruences. With the same method as van der Corput's, Erdős [3] constructed a residue class of odd numbers which contains no integers of the above form. Extending Erdős's argument, Cohen and Selfridge [1] constructed a 26 digit number which is neither the sum nor the difference of two prime powers. Inspired by their work, Z.W. Sun (see 9]) constructed a residue class of odd integers consisting exclusively of numbers not of the form $\pm p^{a} \pm q^{b}$ with some primes $p$ and $q$ and some nonnegative integers $a$ and $b$. We mention that Erdős asked if there exist infinitely many positive integers which are not representable as a sum or difference of two powers (see [5]) and a partial result can be found in [6].

In this paper, we show that there exist infinitely many positive integers which are not of the form $p^{a}+q^{b}$ with primes $p$ and $q$ and nonnegative integers $a$ and $b$ and which further can be chosen to be members of the Fibonacci sequence $\left(F_{n}\right)_{n \geq 0}$ given by $F_{0}=0, F_{1}=1$ and $F_{n+2}=F_{n+1}+F_{n}$ for $n \geq 0$.

In what follows, we use the Vinogradov symbols $\gg$ and $\ll$ with their usual meanings. We recall that given two functions $A$ and $B$ of the real variable $x$, the notations $A \ll B$ and $B \gg A$ are equivalent to the fact that the inequality $|A(x)| \leq c B(x)$ holds with some positive constant $c$ and for all sufficiently large real numbers $x$.

Received by the editors February 13, 2004.

2000 Mathematics Subject Classification. Primary 11B39; Secondary 11B25, 11B50, 11P32.

Key words and phrases. Fibonacci numbers, arithmetic progressions, covering system of congruences.

(C)2005 American Mathematical Society Reverts to public domain 28 years from publication 1887 


\section{The RESUlt}

Our main result is the following.

Theorem 1. There exists a positive integer $n_{0}$ such that if $n>n_{0}$ and

$$
n \equiv 1807873 \quad(\bmod 3543120)
$$

then $F_{n} \neq p^{a}+q^{b}$ with $p$, q prime numbers and $a, b$ nonnegative integers.

We use the same method employed in [2, 3, 9]. However, there are additional difficulties which arise because we want our numbers to belong to the Fibonacci sequence.

Let us quickly recall how one can create a residue class of odd integers most of which are not sums of two prime powers. Well, assume that $n$ is odd and a sum of two prime powers, say $p^{a}+q^{b}$. Since $n$ is odd, it follows that one of $p$ and $q$, say $p$, equals 2. Suppose now that we are given a finite set of triples $\left(a_{i}, b_{i}, p_{i}\right)_{i=1}^{s}$ where $a_{i}$ and $b_{i}$ are nonnegative integers and $p_{i}$ are distinct primes such that the following hold:

(i): for every $a \in \mathbb{Z}$ there exists $i \in\{1, \ldots, s\}$ such that $a \equiv a_{i}\left(\bmod b_{i}\right)$;

(ii): $p_{i} \mid\left(2^{b_{i}}-1\right)$ for all $i=1, \ldots, s$;

We may then choose $n$ to belong to the arithmetic progression given by $n \equiv 2^{a_{i}}$ $\left(\bmod p_{i}\right)$. Since the $p_{i}$ 's are distinct primes, the above system admits a unique solution modulo $p_{1} \ldots p_{s}$ by the Chinese Remainder Lemma. Let $\mathcal{A}$ be this progression. Assume that $n$ is sufficiently large in the above progression $\mathcal{A}$ and that $n=2^{a}+q^{b}$ holds with some prime number $q$. By (i) above, there exists $i \in\{1, \ldots, s\}$ such that $a \equiv a_{i}\left(\bmod b_{i}\right)$. By (ii) above, $2^{a} \equiv 2^{a_{i}}\left(\bmod p_{i}\right)$. However, $n \equiv 2^{a_{i}}\left(\bmod p_{i}\right)$, and therefore $n \equiv 2^{a}\left(\bmod p_{i}\right)$. Hence, $p_{i} \mid\left(n-2^{a}\right)$. However, $n-2^{a}=q^{b}$. Since $q$ is prime, it follows that $p_{i}=q$. Now let $X$ be a very large positive real number. The number of positive integers $n \leq X$ which belong to $\mathcal{A}$ is $\gg X$. However, the number of positive integers of the form $2^{a}+q^{b}$ with $q \in\left\{p_{1}, \ldots, p_{s}\right\}$ and which are $\leq X$ is $\ll \log ^{2} X$. This shows that most numbers in $\mathcal{A}$ are not of the form $p^{a}+q^{b}$.

We now modify the above construction in order to insure that our numbers can be chosen from the Fibonacci sequence. Let $k$ be a positive integer. It is known that $\left(F_{n}\right)_{n \geq 0}$ is periodic modulo $k$. We let $h(k)$ denote this period. Moreover, for integers $f$ and $k$ we write $\mathcal{A}(f, k)$ for the set of residue classes $n$ modulo $h(k)$ such that $F_{n} \equiv f(\bmod k)$.

Assume now that $\left(a_{i}, b_{i}, p_{i}\right)_{i=1}^{s}$ is a finite set of triples of nonnegative integers $a_{i}$ and $b_{i}$ and distinct odd primes $p_{i}$ for $i=1, \ldots, s$ which fulfill the following conditions:

(i): for every $a \in \mathbb{Z}$ there exists $i \in\{1, \ldots, s\}$ such that $a \equiv a_{i}\left(\bmod b_{i}\right)$;

(ii): $p_{i} \mid\left(2^{b_{i}}-1\right)$ for all $i=1, \ldots, s$;

(iii): the set

$$
\bigcap_{i=1}^{s} \mathcal{A}\left(2^{a_{i}}, p_{i}\right) \neq \emptyset .
$$

Moreover, if there exists $i \in\{1, \ldots, s\}$ such that $3 \mid h\left(p_{i}\right)$, then we shall assume that the above intersection contains a class coprime to 3 .

Let $x$ be an element of the set $\bigcap_{i=1}^{s} \mathcal{A}\left(2^{a_{i}}, p_{i}\right)$. Note that $x$ is defined only modulo $M=\operatorname{lcm}\left[h\left(p_{1}\right), \ldots, h\left(p_{s}\right)\right]$. Moreover, if $3 \mid M$, then $x$ is not a multiple of 3 . If $M$ is not a multiple of 3 we replace $M$ by $3 M$ and $x$ by the solution of 
the system of congruences $x(\bmod M)$ and $1(\bmod 3)$. Assume now that $n \equiv x$ $(\bmod M)$ and that $F_{n}=p^{a}+q^{b}$ with some primes $p$ and $q$ and nonnegative integers $a$ and $b$. It then follows that $F_{n}$ is an odd integer, because the only even Fibonacci numbers are those whose indices are multiples of 3 . Since $F_{n}$ is odd, it follows that one of $p$ and $q$, say $p$, is 2 . By (i) above, there exists $i \in\{1, \ldots, s\}$ such that $a \equiv a_{i}$ $\left(\bmod b_{i}\right)$. By (ii) above, it follows that $2^{a} \equiv 2^{a_{i}}\left(\bmod p_{i}\right)$. By the choice of $n$, we have that $F_{n} \equiv 2^{a_{i}}\left(\bmod p_{i}\right)$. Thus, $F_{n} \equiv 2^{a}\left(\bmod p_{i}\right)$. In particular, $p_{i} \mid\left(F_{n}-2^{a}\right)$. However, since $F_{n}-2^{a}=q^{b}$, it follows that $q=p_{i}$. Thus, $q \in\left\{p_{1}, \ldots, p_{s}\right\}$. We now get that $F_{n}=2^{a}+p_{i}^{b}$ for some $i=1, \ldots, s$. Since $F_{n}=\frac{1}{\sqrt{5}}\left(\alpha^{n}-\beta^{n}\right)$, where $\alpha=\frac{1+\sqrt{5}}{2}$ and $\beta=\frac{1-\sqrt{5}}{2}$, we may apply a well-known result from the theory of $\mathcal{S}$-unit equations (see [4) to conclude that such an equation can have only finitely many solutions $(n, a, b)$. Thus, there exists $n_{0}$ with the property that if $n>n_{0}$ and $n \equiv x(\bmod M)$, then $F_{n}$ is not of the form $p^{a}+q^{b}$ with primes $p$ and $q$ and nonnegative integers $a$ and $b$.

In order to finish the proof of the theorem, it suffices to find a finite set of triples $\left(a_{i}, b_{i}, p_{i}\right)_{i=1}^{s}$ fulfilling (i)-(iii) above.

We first note that by taking $s=7$ and

$$
\left(\left(a_{1}, b_{1}\right), \ldots,\left(a_{7}, b_{7}\right)\right)=((0,2),(0,3),(3,4),(1,12),(5,36),(17,36),(29,36))
$$

we get that (i) above is fulfilled. Indeed, every integer is congruent either to 0 $(\bmod 2)$ or to $0(\bmod 3)$ or to $3(\bmod 4)$ or to $1(\bmod 12)$ or to $5(\bmod 12)$, and in this last case it is congruent to either 5,17 or 29 modulo 36 . We now take $\left(p_{1}, \ldots, p_{7}\right)=(3,7,5,13,19,37,73)$ and note that condition (ii) above is fulfilled. It is easy to check that $\left(h\left(p_{1}\right), \ldots, h\left(p_{7}\right)\right)=(8,16,20,28,18,76,148)$. Finally, it is easy to see that

$$
\begin{aligned}
& \mathcal{A}\left(2^{a_{1}}, p_{1}\right)=\mathcal{A}\left(2^{0}, 3\right)=\{1,2,7\} \quad(\bmod 8), \\
& \mathcal{A}\left(2^{a_{2}}, p_{2}\right)=\mathcal{A}\left(2^{0}, 7\right)=\{1,2,6,15\} \quad(\bmod 16), \\
& \mathcal{A}\left(2^{a_{3}}, p_{3}\right)=\mathcal{A}\left(2^{3}, 5\right)=\{4,6,7,13\} \quad(\bmod 20), \\
& \mathcal{A}\left(2^{a_{4}}, p_{4}\right)=\mathcal{A}\left(2^{1}, 13\right)=\{3,25\} \quad(\bmod 28), \\
& \mathcal{A}\left(2^{a_{5}}, p_{5}\right)=\mathcal{A}\left(2^{5}, 19\right)=\{7,11\} \quad(\bmod 18), \\
& \mathcal{A}\left(2^{a_{6}}, p_{6}\right)=\mathcal{A}\left(2^{17}, 37\right)=\{10,15,28,61\} \quad(\bmod 76), \\
& \mathcal{A}\left(2^{a_{7}}, p_{7}\right)=\mathcal{A}\left(2^{29}, 73\right)=\{53,95\} \quad(\bmod 148) .
\end{aligned}
$$

One now checks that the system of congruences $x \equiv 1(\bmod 8), x \equiv 1(\bmod 16)$, $x \equiv 13(\bmod 20), x \equiv 25(\bmod 28), x \equiv 7(\bmod 18), x \equiv 61(\bmod 76)$ and $x \equiv 53(\bmod 148)$ has a positive integer solution $x$. In fact, the above system is equivalent to the system of congruences $x \equiv 1(\bmod 16), x \equiv 3(\bmod 5), x \equiv$ $4(\bmod 7), x \equiv 7(\bmod 9), x \equiv 4(\bmod 19)$ and $x \equiv 16(\bmod 37) . \quad$ Solving this system, we get $x \equiv 1807873(\bmod 3543120)$, which, together with the above arguments completes the proof of Theorem 1.

We would like to conclude by offering the following problem.

Problem. Find an arithmetic progression of positive integers $A(\bmod B)$ such that if $n>n_{0}$ satisfies $n \equiv A(\bmod B)$, then $F_{n} \neq \pm p^{a} \pm q^{b}$ for any primes $p, q$ and nonnegative integers $a, b$. 


\section{ACKNOWLEDGMENTS}

This work was done during a visit of F. L. at the Mathematics Department of the Auburn University at Montgomery. He thanks the people of this department for their warm hospitality.

\section{REFERENCES}

[1] F. Cohen and J. L. Selfridge, 'Not every number is the sum or difference of two prime powers', Math. Comp. 29 (1975), 79-81. MR0376583 (51:12758)

[2] J. G. van der Corput, 'On de Polignac's conjecture', Simon Stevin 27 (1950), 99-105. MR0035298 (11:714e)

[3] P. Erdős, 'On integers of the form $2^{k}+p$ and some related problems', Summa Brasil. Math. 2 (1950), 113-123. MR0044558 (13:437i)

[4] J.-H. Evertse, K. Győry, C. L. Stewart and R. Tijdeman, ' $S$-unit equations and their applications', in New advances in transcendence theory (Durham, 1986), 110-174, Cambridge Univ. Press, Cambridge, 1988. MR0971998|(89j:11028)

[5] R. K. Guy, Unsolved Problems in Number Theory, Springer, 1994. MR.1299330 (96e:11002)

[6] F. Luca, 'On integers which are not differences of two powers', Mathematika, 49 (2002), no. 1-2. 231-243 (2004). MR2059057 (2004c:11041)

[7] A. de Polignac, 'Recherches nouvelles sur les nombres premiers', C.R. Acad. Sci. Paris Math. 29 (1949), 397-401, 738-739.

[8] N. P. Romanoff, 'Über einige Sätze der additiven Zahlentheorie ', Math. Ann. 57 (1934), 668-678.

[9] Z. W. Sun, 'On integers not of the form $\pm p^{a} \pm q^{b}$, Proc. Amer. Math. Soc. 128 (2000), 997-1002. MR 1695111 (2000i:11157)

imate, Unam, Ap. Postal 61-3 (Xangari), CP. 58 089, Morelia, Michoacán, Mexico E-mail address: fluca@matmor.unam.mx

Department of Mathematics, Auburn University Montgomery, Montgomery, Alabama 36124-4023

E-mail address: pstanica@mail.aum.edu 